\title{
CONTRIBUIÇÃO AO ESTUDO DA HIPSARRITMIA
}

\author{
ANa Maria Fisch * \\ Clovis Oliveira** \\ ISMAR FERNANDES ***
}

Assunto dos mais interessantes, a hipsarritmia é ainda pouco conhecida em sua etiopatogenia. Em 1841, West publicou a primeira descrição clínica de uma forma singular de epilepsia, caracterizada pela flexão da cabeça, reviramento dos olhos e perda da consciência, em criança com acentuado retardamento motor. Em 1849, Newham, citado por Bower e Jeavons ${ }^{1}$, descreveu o quadro clínico característico dos espasmos em flexão, chamando a atenção para o fato dos pacientes serem retardados mentais. Em 1925, Azal e Moro, segundo Bower e Jeavons ${ }^{1}$, denominaram a sindrome de "Blitzkrämpfen" e "Salaamkrämpfen".

Gibbs e Gibbs ${ }^{8}$, em 1952, destacaram um tipo particular de traçado eletrencefalográfico que denominaram de hipsarritmia (de hipsus - altura), em cuja sintomatologia correspondia aos espasmos em flexão da criança. A expressão fêz fortuna e logo despertou grande interêsse, com avultado número de publicações a respeito. Hess e Neuhaus ${ }^{10}$, em 1952, também descreveram êsses aspectos eletrencefalográficos, relacionando-os às crises de "Blitz, Nick e Salaamkrämpfen". Em 1953, Gastaut e Roger ${ }^{6}$ designaram de "dysrythmie majeure" o aspecto eletrencefalográfico característico dos espasmos em flexão. Druckman e Chao ${ }^{4}$, em 1955, caracterizaram um tipo de crise que chamaram de "espasmos em massa", consistente na súbita e intensa contração da maior parte da musculatura corporal, geralmente com flexão e adução dos membros, como a dobrar o corpo; tais ataques assemelhavam-se, no dizer dos próprios autores, ao reflexo de Moro exagerado; outros, menos freqüentes, eram em extensão, a lembrarem os fenômenos de rigidez de descerebração.

Não é difícil entender que, com variações de pequena monta, cuidaram os autores citados, da mesma síndrome, à qual se ajustou o aspecto eletrográfico peculiar.

Em 1954, Gibbs, Fleming e Gibbs ${ }^{9}$ acrescentaram notável contribuição, com uma casuística de 237 pacientes, estabelecendo as necessárias correla-

Trabalho realizado no Instituto de Neurologia da Universidade do Brasil (Diretor: Prof. Deolindo Couto), apresentado ao I Congresso Brasileiro de Neurologia (Ribeirão Prêto, SP, em julho - 1964); * Assistente do Instituto de Neurologia; ** Docente livre da Fac. Med. da Univ. do Brasil e da Fac. Med. da Univ. Fed. do Estado do Rio de Janeiro e Assistente do Instituto de Neurologia; *** Professor Adjunto da Fac. Med. da Univ. do Brasil e Assistente do Instituto de Neurologia. 
ções clínico-eletrográficas. Outros trabalhos foram, a seguir, cuidando dos vários aspectos que a curiosa forma de epilepsia revelava. Stamps, Gibbs e Haase 24 foram os primeiros a empregar os antibióticos na terapêtica; Sorel e col. ${ }^{23}$ introduziram o tratamento pelo ACTH; Passouant e col. ${ }^{18}$ relacionaram a hipsarritmia ao problema da maturação cerebral; Kellaway ${ }^{11}$, baseado em apreciável casuística, ocupou-se da sintomatologia e da interpretação etiopatogênica, chamando a atenção para o papel da maturação cerebral.

Em nosso país, Oliveira e Fernandes ${ }^{17}$, em 1961, a propósito de 8 casos, foram os primeiros a tratar do assunto, em trabalho no qual foi feita a revisão da matéria, em especial no que respeita à sintomatologia, à etiopatogenia e ao caráter evolutivo do traçado hipsarrítmico. Maia e Lins ${ }^{15}$, também em 1961, publicaram trabalho no qual, ao lado da apreciação clínica e eletrográfica, apresentaram interessantes dados sôbre os resultados terapêuticos. O tema voltou a ser ventilado, em 1963, em comunicação à I Reunião da Academia Brasileira de Neurologia, por Nery e Mello ${ }^{16}$.

Conceito - Hipsarritmia é sindrome própria da primeira infância que se caracteriza, sob o aspecto clínico, por fenômenos episódicos, traduzidos principalmente pelos espasmos em flexão, mas também por convulsões ou cutra manifestação nervosa de feitio variável, associadas a retardamento mental e, não raro, também motor. Eletrencefalogràficamente, manifesta-se por extrema anormalidade do traçado, onde se vêem ondas lentas e pontas de alta voltagem, desordenadamente, variando as pontas em duração e localização, ora parecendo focais em um ponto e logo dando a impressão de se originarem de outro ou de múltiplos focos. A descarga de pontas, às vêzes, é generalizada, porém, nunca rìtmicamente repetida nem organizada como no pequeno mal ou na sua variante (Gibbs e Gibbs ${ }^{8}$ ). A disritmia é pràticamente contínua, sem se modificar na vigília ou no sono, nem com a foto-estimulação (Gastaut e Roger ${ }^{6}$ ). A reação de parada é nula (Thiebaut e col. $\left.{ }^{26}\right)$. A própria crise clínica pouco altera o traçado; quando o faz, ora provoca descargas de ondas rápidas e pontas de alta voltagem pós-crítica (Gibbs, Fleming e Gibbs ${ }^{9}$; Thiebaut e col. ${ }^{26}$ ), ora fase de depressão ou supressão da atividade elétrica durante o ataque, a qual pode persistir por curto período pós-crítico (Kellaway ${ }^{11}$ ).

Etiopatogenia - Enquanto a sintomatologia e o aspecto eletrencefalográfico são bem conhecidos, a etiopatogenia da hipsarritmia não está ainda estabelecida. Sabe-se que a disritmia se desenvolve em cérebro imaturo. Não se pode entender a hipsarritmia sob um plano estático. Ao contrário, ela parece traduzir um fenômeno essencialmente dinâmico, evolutivo. É preciso, como disse Kellaway ${ }^{11}$, conhecer sua história natural para poder situar bem o problema. Importa, então, saber a época em que se instala a anomalia, em que sentido evolve e quais as modificações eletrográficas e clínicas que provoca. Importa, também, conhecer se se trata de criança prèviamente sadia ou já enfêrma. E é esta essência do fenômeno, a natureza do processo, como e porque se instala, inclusive em cérebros aparentemente 
diferentes quanto ao seu estado de higidez precedente, que continuamos a desconhecer.

Gastaut e Roger ${ }^{6}$ e Kellaway ${ }^{11}$ já frisaram que a evolução do quadro eletroclínico se baseia na época em que o cérebro sofre as influências anormais e não na natureza do insulto cerebral. A hipsarritmia instala-se ou surge na criança de até 3 anos de idade, especialmente no $10^{\circ}$ ano e, mais precisamente, nos primeiros 6 meses de vida (Chao, Druckman e Kellaway ${ }^{2}$ ). Gibbs e Gibbs ${ }^{8}$ referem-se a dois pacientes com mais de 7 anos, chamando a atenção para a sua raridade. Kellaway ${ }^{11}$ afirma que, em sua experiência, - traçado hipsarrítmico nunca resultou de distúrbio cerebral ocorrido depois do segundo ano de vida; observou, durante mais de 10 anos, grande número de crianças com encefalite, acidentes traumáticos encefálicos e outras lesões cerebrais; no particular dos traumatismos, acompanhou mais de 500 crianças gravemente atingidas nas várias idades durante muitos anos, e registrou diversos casos, porém nenhum no qual o trauma tivesse ocorrido após a idade de 30 meses.

interessante anotar que, em cêrca da metade das estatísticas, tem sido impossivel precisar a causa. Tal ocorreu em $55 \%$ dos casos de Gibbs e Gibbs ${ }^{8}$ e em $42 \%$ dos de Kellaway ${ }^{11}$. Sem entrar no mérito das sistematizações, podemos dizer que, na etiologia da hipsarritmia, comparecem as mesmas condições que entram na gênese das encefalopatias da infância. $\hat{\mathbf{E}}$ compreensivel que, sendo a hipsarritmia de maior incidência na primeira infância, sejam as nóxias que mais cedo aparecem, as responsáveis por maior número de casos. $\mathrm{E}$ aqui têm particular importância os fatôres genéticos e os traumatismos pré-natais e natais, a que se seguem as infecções verificadas na primeira infância. Em nosso material, 7 pacientes tinham história de nascimento mediante parto laborioso, com anóxia, dos quais 3 foram extraídos a fórceps; em 4 pacientes as manifestações clínicas surgiram na vigência de ou em seguida a episódios febris; um dêles foi acometido de poliomielite aos 3 meses de idade; em 8 casos não conseguimos apurar qual o agente causador das crises.

Os traumatismos pré-natais ou relacionados ao nascimento são comuns na gênese das encefalopatias infantis. Conquanto a ação contudente direta sôbre o cérebro seja freqüente, é provável que, em muitos casos, os traumas pequenos ou inaparentes respondam pelo sofrimento encefálico que se manifesta principalmente à custa de deficiência circulatória e respiratória. A anóxia cerebral é de máxima importância, dada a extrema sensibilidade do tecido nervoso à ausência de oxigênio. E claro que a anóxia pode depender de outros fatôres não traumáticos e variar de intensidade e duração. Representa, a anóxia pré-natal, $20 \%$ das causas arroladas na casuística de Kellaway ${ }^{11}$.

Ao problema de hipotética substância química, possivelmente enzimática, que influencie o comportamento biológico da atividade cerebral, vêm sendo dedicadas muitas pesquisas. Muito pouco ainda se conhece sôbre a essência do fenômeno da maturação cerebral. Roberts ${ }^{20}$, em 1960, afirmava que, em estimativa grosseira, eram conhecidas $10 \%$ das substâncias presentes na célula viva, o que já representava um grande progresso. Não obstante, cha- 
mava a atenção para a importância do metabolismo do ácido gama-aminobutírico (GABA) no cérebro em desenvolvimento. Direta ou indiretamente, esta substância cujo metabolismo é complexo, se relaciona aos processos de inibição.

Sintomatologia - A hipsarritmia deve ser considerada como sindrome eletroclínica. Assim, a sintomatologia bem que sugestiva, não autoriza o diagnóstico, se não confirmada pelo traçado eletrencefalográfico.

Os sintomas mais característicos são os espasmos em flexão da primeira infância, consistentes em contrações súbitas e violentas da musculatura axial ou de todo o corpo, com rápida flexão ou, mais raramente, em extensão, podendo a cabeça tocar os pés, se intensa a flexão, e reviramento dos olhos para cima. São os "massive spasms" de Druckman e Chao ${ }^{4}$, o que corresponde ao reflexo de Moro exagerado. A denominação de "espasmos" é apropriada, pois que tais movimentos são rápidos e fugazes. O estado de consciência nem sempre pode ser apreciado, dada a duração mínima das crises. É provável que, na maioria, haja perda momentânea da consciência. A freqüência é muito variável, podendo ocorrer centenas de crises diárias. Os autores são unânimes em assinalar o alto percentual de retardamento mental, em geral grande. Isso se verifica aqui mais que em qualquer outro tipo de epilepsia, o que fala em favor da hipótese de desenvolvimento do processo hipsarrítmico em cérebro imaturo.

Dois pontos devem merecer atenção especial. A apreciação clínica de um espasmos em flexão, se não intenso, pode passar despercebida. Pode ser confundido, especialmente se o chôro é o elemento a despertar a atenção, com manha, hábito, cólica ou outra intercorrência, conforme referiu Kellaway. O outro aspecto importante é que, embora os espasmos em flexão sejam dominantes, qualquer outro tipo de manifestação epiléptica pode ser o sintoma principal, ou único. Crises do tipo grande mal foram presentes em 33\% dos 237 casos de Gibbs, Fleming e Gibbs ${ }^{9}$. Em nossos casos verificamos $8(40 \%)$ com espasmos em flexão e $10(50 \%)$ com crises de grande mal.

Dos sinais neurológicos merece destaque o retardamento motor, seja à conta de paralisia, seja à conta de alteração do tono, encontrada na maioria dos nossos pacientes. Aliás, deve ser lembrada, também aqui, a dificuldade por vêzes encontrada em apreciar bem êsses dados em recém-nascidos e em crianças muito pequenas (Kellaway ${ }^{11}$ ). É claro que o déficit motor varia em função do quadro clínico apresentado, sendo mais freqüentes as hemi e as paraplegias. Alterações de tono muscular, para mais ou para menos, são comuns nos pacientes com alteração motora. Podem também ocorrer hipercinesias, tal como a atetose. As perturbações da linguagem são importantes, tanto por defeito da palavra pròpriamente, como em conseqüência de acentuado retardamento mental.

Outros distúrbios, como paralisias oculares, nistagmo, alterações da audição, hidrocefalia (caso de Thiebaut ${ }^{25}$ ), catarata congênita (Oliveira e Fernandes ${ }^{17}$ ), têm sido registrados. 
Evolução - O problema da hipsarritmia não pode ser bem compreendido nem a sua exposição será completa se não ajuntarmos a idéia de sua evolução porque a síndrome é, em geral, transitória, com duração variável. Seria, em muitos casos, apenas uma fase, ao menos quanto ao aspecto eletrográfico, de uma encefalopatia ou epilepsia.

Em trabalho anterior 17 já destacávamos êste aspecto curioso e peculiar, dando ênfase àquilo que Kellaway ${ }^{11}$ chamou de história natural da hipsarritmia. Quando se tem a oportunidade de obter eletrencefalogramas muito precoces, êles podem registrar assincronias entre regiões homólogas e descargas polimorfas focais irregulares, antes de se instalar o quadro hipsarritmico. Tal pode ser obtido especialmente no primeiro mês de vida. Re-
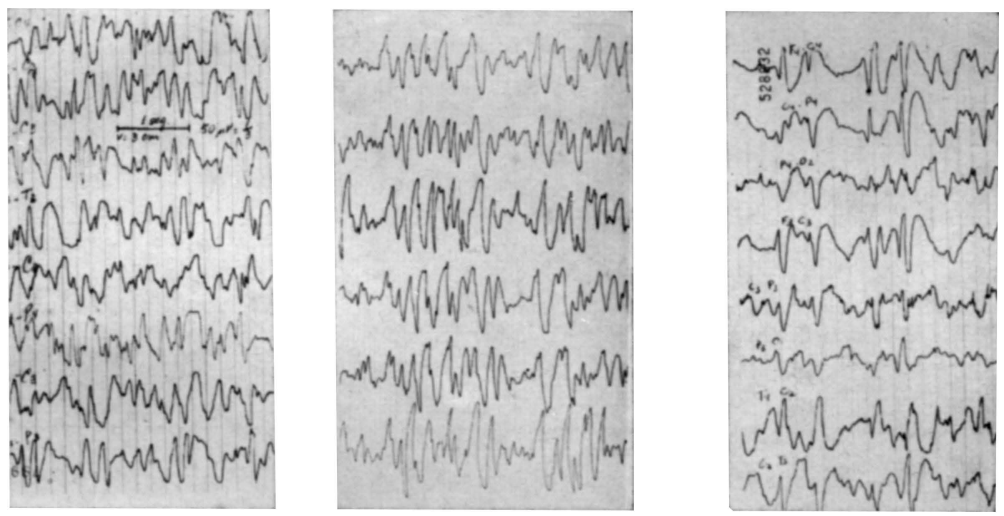

Fig. 1 - Aspectos de tracados hipsarritmicos, observando-se dominancia de pontas de distribuicão difusa.
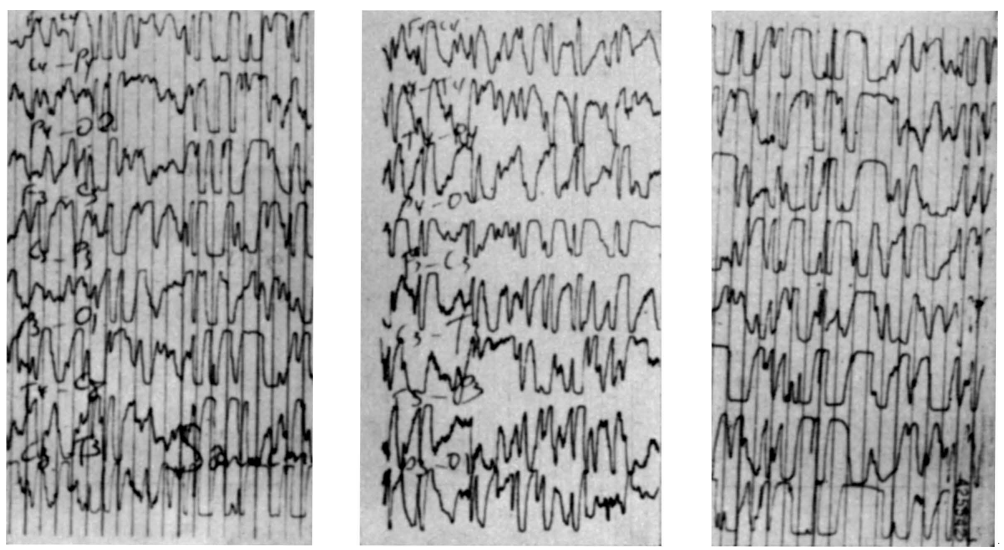

Fig, 2 - Aspectos de traçados hipsarritmicos, observando-se atividade lenta de alta voltagem e complexo ponta-onda degradado. 
gistros subseqüentes revelam a instalação da hipsarritmia com tôdas as características.

Manifestada a hipsarritmia, teremos nova fase evolutiva, que pode ser apreciada pelas modificações eletrográficas, acompanhadas, ou não, pelas manifestações clínicas. Gibbs, Fleming e Gibbs ${ }^{19}$, em 1954, assinalaram que, com a idade, o eletrencefalograma tende a se tornar normal ou a apresentar anomalias focais. Samson-Dollfus ${ }^{22}$, em 1958, estudou os diversos aspectos eletroclínicos relacionados aos vários cursos de sua evolução. Livingston e
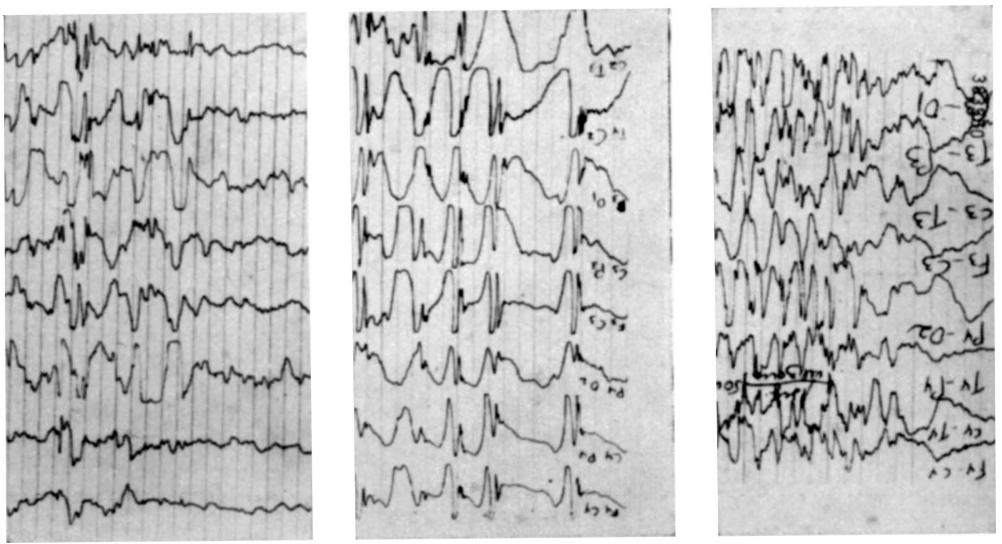

Fig. 3 - Hipsarritmia; zonas de depressão seguidas de surtos de atividade irregular de alta voltagem $e$ pontas isoladas de distribuição difusa.
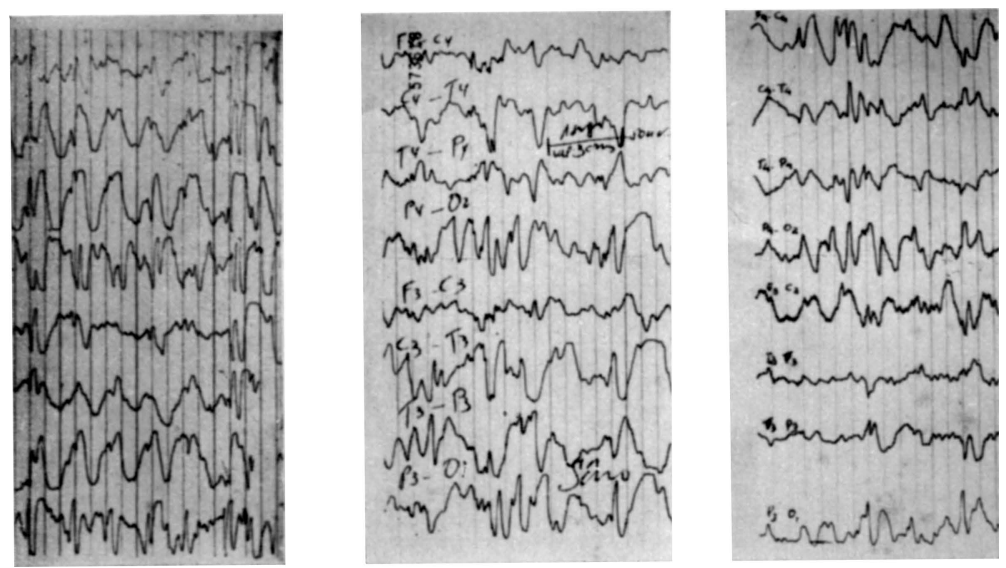

Fig. 4 - $\boldsymbol{A}$ esquerda e no centro, aspectos de traçados hipsarritmicos em sono induzido, sem modificação do ritmo dominante; à direita, alteraçōes difusas com certa depressão no hemisfério esquerdo, revelando assimetria. 
col. ${ }^{13}$ mencionaram a elevada taxa de $58 \%$ de normalização subseqüente de traçado.

A evolução dá-se, em resumo, nos seguintes sentidos: 1) normalização espontânea de traçado, em geral acompanhada de evolução clínica favorável, o que constitui o tipo menos comumente verificado; 2) transformação em traçado focal, com o aparecimento do ritmo de base, sendo os focos variáveis, podendo ser múltiplos, em um ou ambos os hemisférios; 3) anormalidade difusa, porém de caráter diferente (ondas rápidas e de alta voltagem no sono, atividade lenta e paroxística na vigília, ou no desenvolvimento de um tipo monorritmico em vigília com ritmo de base da ordem de $6 \mathrm{c} / \mathrm{s}$, ou ainda, a transformação em um tipo aproximado do pequeno mal variante de Gibbs, traduzido por grupos melhor organizados de polipontas e ondas lentas); 4) finalmente, pode persistir o traçado hipsarritmico.

Patologia - Este capítulo é pouco conhecido, dado o reduzido número de casos autopsiados. O subsidio que podemos apresentar refere-se ao caso 19, no qual a necropsia revelou edema cerebral, hiperemia dos vasos da leptomeninge, fibrose da leptomeninge com aderếncia meningocortical no lobo frontal esquerdo e lesão isquêmica do complexo ventral do tálamo direito.

Tratamento - Este aspecto tem sido amplamente cuidado sem, contudo, apresentar conclusões positivas. Isto resulta, parece, da diversidade de fatôres e causas responsáveis pelo desencadeamento da hipsarritmia.

Após os tratamentos iniciais com anticonvulsivantes, quase sempre falhos, várias associações terapêuticas foram propostas. Em 1951, Stamps, Gibbs e Haase ${ }^{25}$ empregaram antibióticos (aureomicina, terramicina e cloromicetina) em altas doses, com bons resultados. Em 1959, Sorel e col. ${ }^{23}$ publicaram os primeiros resultados com o emprêgo do ACTH, sendo o bom êxito dessa terapêutica confirmado por vários autores. Emprega-se hoje, em especial, o hormônio de ação retardada. Para Sorel ${ }^{23}$ os resultados são condicionados às seguintes normais: a) quando o tratamento é iniciado entre o $2 .^{\circ}$ e $5 .^{\circ}$ dias de doença há regressão de todos os sintomas; b) quando o tratamento é iniciado após 3 anos de enfermidade, os resultados podem ser satisfatórios no que diz respeito à ausência de espasmos e alterações elétricas, porém o retardamento mental fica permanente. Em resumo, conclui aquêle autor, os resultados parecem melhores se a criança gozava de perfeita saúde antes, se o ACTH foi empregado no primeiro mês de evolução e se o EEG se regularizou sem anormalidade elétrica focal.

Não nos parece lícito argumentar com pequena experiência pessoal, inclusive porque vários de nossos pacientes já haviam recebido orientação terapêutica anterior, perturbando, em parte, a correta apreciação dos resultados. Entendemos, todavia, que, em apreciável número de casos, retornam os sintomas logo que supressa a medicação adrenocorticotrópica, o que tem sido registrado por diversos autores. Por outro lado, temos observado a instalação da síndrome de Cushing, embora transitória, em alguns casos, o que limita o emprêgo terapêutico do hormônio em questão. 


\begin{tabular}{|c|c|c|c|c|c|}
\hline Casos & & $\begin{array}{l}\text { Idade } \\
\text { exo }- \text { Côr }\end{array}$ & $\begin{array}{l}\text { Idade do } \\
\text { intcio }\end{array}$ & Antecedentes & Sintomas principais \\
\hline$\stackrel{1}{\text { MASL }}$ & & $\begin{array}{l}\text { anos } \\
\text { f. } \\
\text { branca }\end{array}$ & 3 meses & Parto distócico (fórcipe) & $\begin{array}{l}\text { Espasmos em flexão da ca- } \\
\text { beça e reviramento dos } \\
\text { olhos com perda da cons- } \\
\text { ciência }\end{array}$ \\
\hline$\stackrel{2}{L F L}$ & & $\begin{array}{l}\text { meses } \\
\text { f. } \\
\text { branca }\end{array}$ & 1 mês & $\begin{array}{l}\text { Sindrome febril com exci- } \\
\text { tação psicomotora e in- } \\
\text { sônia, precedendo a do- } \\
\text { ença }\end{array}$ & $\begin{array}{l}\text { Espasmos em extensão da } \\
\text { cabeça com flexão dos } \\
\text { membros }\end{array}$ \\
\hline$\stackrel{3}{\mathrm{~J}} \mathrm{LZ}$ & 22 & $\begin{array}{l}\text { meses } \\
\text { m. } \\
\text { branca }\end{array}$ & 8 meses & Discreto retardo motor & $\begin{array}{l}\text { Espasmos em flexão da ca- } \\
\text { beça e dos membros su- } \\
\text { periores }\end{array}$ \\
\hline$\stackrel{4}{\mathrm{FF}}$ & 3 & $\begin{array}{l}\text { anos } \\
\text { m. } \\
\text { preta }\end{array}$ & 3 anos & Nascido a fórcipe & $\begin{array}{l}\text { Espasmos em flexão da ca- } \\
\text { beca }\end{array}$ \\
\hline$\stackrel{5}{\mathrm{LHA}}$ & 4 & $\begin{array}{l}\text { anos } \\
\text { m. } \\
\text { branca }\end{array}$ & 30 meses & $\begin{array}{l}\text { Parto demorado; morte } \\
\text { aparente }\end{array}$ & $\begin{array}{l}\text { Crises convulsivas genera- } \\
\text { lizadas }\end{array}$ \\
\hline$\stackrel{6}{\text { MAM }}$ & 14 & $\begin{array}{l}\text { meses } \\
\text { m. } \\
\text { branca }\end{array}$ & 10 meses & $\begin{array}{l}\text { Parto demorado; morte } \\
\text { aparente }\end{array}$ & $\begin{array}{l}\text { Crises convulsivas genera- } \\
\text { lizadas }\end{array}$ \\
\hline$\stackrel{7}{R G}$ & 6 & $\begin{array}{l}\text { anos } \\
\text { f. } \\
\text { branca }\end{array}$ & 17 meses & $\begin{array}{l}\text { Sindrome febril aos } 17 \text { me- } \\
\text { ses, após a qual não } \\
\text { mais falou nem andou }\end{array}$ & $\begin{array}{l}\text { Flexão da cabeça e queda } \\
\text { ao solo, dizendo pala- } \\
\text { vras desconexas; crises } \\
\text { convulsivas }\end{array}$ \\
\hline$\stackrel{8}{\text { WPS }}$ & & $\begin{array}{l}\text { anos } \\
\text { m. } \\
\text { preta }\end{array}$ & 18 meses & 一 & $\begin{array}{l}\text { Crises convulsivas genera- } \\
\text { lizadas; excitação psico- } \\
\text { motora intercritica }\end{array}$ \\
\hline$\stackrel{9}{\mathrm{DAP}}$ & 3 & $\begin{array}{l}\text { anos } \\
\text { m. }\end{array}$ & 18 meses & 一 & $\begin{array}{l}\text { Crises convulsivas genera- } \\
\text { lizadas }\end{array}$ \\
\hline$\stackrel{10}{\text { VPA }}$ & 9 & $\begin{array}{l}\text { meses } \\
\text { f. } \\
\text { branca }\end{array}$ & 5 meses & - & $\begin{array}{l}\text { Espasmos em flexão da ca- } \\
\text { beça }\end{array}$ \\
\hline$\stackrel{11}{\mathrm{MCR}}$ & 26 & $\begin{array}{l}\text { meses } \\
\text { f. } \\
\text { branca }\end{array}$ & 26 meses & - & $\begin{array}{l}\text { Crises de flexão da cabeça } \\
\text { com perda da consciên- } \\
\text { cia }\end{array}$ \\
\hline $\begin{array}{r}12 \\
\text { AR }\end{array}$ & 23 & $\begin{array}{l}\text { meses } \\
\text { m. } \\
\text { branca }\end{array}$ & 11 meses & $\begin{array}{l}\text { Deixou de andar aos } 20 \\
\text { meses }\end{array}$ & $\begin{array}{l}\text { Espasmos e crises tônicas } \\
\text { generalizadas com perda } \\
\text { da consciência }\end{array}$ \\
\hline $\begin{array}{l}13 \\
\text { MEA }\end{array}$ & 5 & $\begin{array}{l}\text { anos } \\
\text { f. } \\
\text { branca }\end{array}$ & 4 anos & Doença febril aos 3 meses & $\begin{array}{l}\text { Torção da cabeça e con- } \\
\text { vulsões generalizadas }\end{array}$ \\
\hline $\begin{array}{l}14 \\
\text { RMF }\end{array}$ & 19 & $\begin{array}{l}\text { meses } \\
\text { m. } \\
\text { branca }\end{array}$ & 19 meses & $\begin{array}{l}\text { Vários familiares com epi- } \\
\text { lepsia e doença mental }\end{array}$ & $\begin{array}{l}\text { Crises acinéticas e convul- } \\
\text { sões generalizadas }\end{array}$ \\
\hline $\begin{array}{l}15 \\
\text { IRS }\end{array}$ & 12 & $\begin{array}{l}\text { meses } \\
\text { m. } \\
\text { branca }\end{array}$ & 5 meses & - & $\begin{array}{l}\text { Espasmos localizados ou } \\
\text { generalizados e ausên- } \\
\text { cias }\end{array}$ \\
\hline$\stackrel{16}{\mathrm{CL}}$ & 18 & $\begin{array}{l}\text { meses } \\
\text { m. } \\
\text { branca }\end{array}$ & 17 meses & 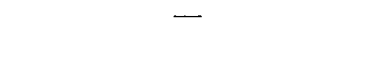 & $\begin{array}{l}\text { Espasmos localizados ou } \\
\text { generalizados e convul- } \\
\text { sōes generalizadas }\end{array}$ \\
\hline $\begin{array}{l}17 \\
\text { SS }\end{array}$ & 7 & $\begin{array}{l}\text { anos } \\
\text { m. } \\
\text { branca }\end{array}$ & 3 meses & $\begin{array}{l}\text { Sindrome febril preceden- } \\
\text { do as crises }\end{array}$ & $\begin{array}{l}\text { Espasmos localizados ou } \\
\text { generalizados, com per- } \\
\text { da da consciência }\end{array}$ \\
\hline $\begin{array}{ll}18 \\
\text { LRA }\end{array}$ & 5 & $\begin{array}{l}\text { anos } \\
\text { m. } \\
\text { branca }\end{array}$ & 2 anos & $\begin{array}{l}\text { A mãe teve "ameaço" de } \\
\text { abôrto no } 4 \circ \text { mês de ges- } \\
\text { tação }\end{array}$ & $\begin{array}{l}\text { Convulsões generalizadas; } \\
\text { crises de palidez com } \\
\text { cianose }\end{array}$ \\
\hline$\stackrel{19}{\text { FMA }}$ & 3 & $\begin{array}{l}\text { anos } \\
\text { m. } \\
\text { branca }\end{array}$ & 9 meses & $\begin{array}{l}\text { Parto cesáreo. Andou com } \\
11 \text { meses. Falou com } 2 \\
\text { anos }\end{array}$ & $\begin{array}{l}\text { Espasmos em flexão. Cri- } \\
\text { ses jacksonianas à es- } \\
\text { querda, Convulsões ge- } \\
\text { neralizadas. Mioclonias }\end{array}$ \\
\hline $\begin{array}{c}20 \\
\text { LSL }\end{array}$ & & $\begin{array}{l}\text { anos } \\
\text { f. } \\
\text { preta }\end{array}$ & 7 anos & Mãe débil mental & $\begin{array}{l}\text { Espasmos nos membros di- } \\
\text { reitos e contrações ge- } \\
\text { neralizadas, com perda } \\
\text { da consciência }\end{array}$ \\
\hline
\end{tabular}


Hipotonia e hiporreflexia. Grande retardo motor; não fica de pé nem senta

Normal

Paraplegia flácida

Hipotonia e hiporreflexia

Ncrmal

Grande retardo motor; ainda não senta

Grande retardo motor. Não anda nem fala

Bom desenvolvimento motor; ainda não fala

Grande atraso motor

Microcéfalo, epicantus, hipertelorismo, hipotonia, hiporreflexia

Hipotonia

Hipotonia e hiporreflexia. Miose em AO

Normal

Não anda nem fala

Normal

Hipotonia; não sustenta a cabeça nem senta. Amaurose

Normal

Normal

Hemiplegia à esquerda

Catarata congênita em AO e amaurose. Não fala. Hiporreflexia

\section{Retardo}

Oligofrenia IIT

Péssima

Agitação psicomotora

Má

Oligofrenia III

Oligofrenia III

Oligofrenia III

Debilidade

Debilidade

Retardo

Oligofrenia III

\section{Oligofrenia III}

Retardo

$-$

Retardo

Não apreciável

Oligofrenia II

Retardo

Debilidade, impulsividade, agressividade

Deterioração progressiva

Retardo
Má

Razoável

Má

Péssima

Má

Má
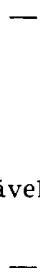

Deixou de andar

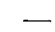
Contrôle d a s crises
Contrôle
crises
Contrôle par- cial das cri- ses

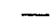

Muito boa. MeIhora eletrográfica

Má

Má
Anticonvulsivantes, GABA, corticosteróides

Anticonvulsivantes

Anticonvulsivantes ACTH, corticosteróides tranquilizantes, GABA

Anticonvulsivantes, ACTH, corticosteróides,

Anticonvulsivantes, ACTH, corticosteróides

Anticonvulsivantes

Anticonvulsivantes e tranqüilizantes

Tranqüilizantes e anticonvulsivantes

Anticonvulsivantes e AAS

Anticonvulsivantes, AAS e GABA

Anticonvulsivantes

Anticonvulsivantes

Abandonado

Anticonvulsivantes

Antibióticos

Corticosteróides, anticonvulsivantes, ácido glutâmico, antibióticos Anticonvulsivantes

\section{Anticonvulsivantes}

Anticonvulsivantes, antibióticos, ACTH, corticosteróides

Anticonvulsivantes 


\section{CASUISTICA}

Apresentamos 20 casos de hipsarritmia, eletrencefalogràficamente diagnosticados. E compreensivel que nosso material, conquanto pequeno, difira do que é registrado em serviços pediátricos, para os quais se dirige o grande contingente dos pequenos enfermos. Ao nosso Serviço de Clínica Neurológica, vêm as crianças, em geral, encaminhadas por outras clínicas, sendo natural que o material não se preste à análise estatística no que se refere ao fator etário. Os principais dados relativos aos 20 casos estão sumariados no quadro anexo.

Quanto à idade, um paciente tinha menos de 6 meses, 2 tinham de 7 a 12 meses, 2 tinham de 13 a 18 meses, 4 tinham de 19 a 24 meses e 11 tinham acima de 2 anos. No que respeita à duração da doença, em 5 pacientes $o$ inicio ocorrera entre 1 a 6 meses, em 4 ocorrera entre 7 a 12 meses, em 4 ocorrera entre 13 e 18 meses, em 2 ocorrera entre 19 e 24 meses, em 5 ocorrera mais de 2 anos antes do nosso exame.

Quanto ao tipo de crises clínicas, 8 pacientes $(40 \%)$ apresentavam espasmos em flexão, $10(50 \%)$ apresentavam convulsões tipo grande mal e 2 (10\%) apresentavam crises tipo pequeno mal. A inteligência era normal em um paciente; em 17 havia retardo mental, discreto em $7(35 \%)$ e severo em $10(50 \%)$; em 2 pacientes não pôde ser determinado o desenvolvimento intelectual. O desenvolvimento motor era normal em 6 pacientes; em 13 havia retardo motor, considerado ligeiro em um $(5 \%)$ e severo em $12(60 \%)$; em um paciente não foi determinado o desenvolvimento motor.

\section{CONCLUSOES}

Hipsarritmia não é entidade nosológica, devendo ser considerada como sindrome clínico-eletrográfica e, portanto, como tipo especial de epilepsia, própria da primeira infância.

Só o traçado eletrencefalográfico característico autoriza o rótulo diagnóstico.

No quadro clínico, dominam os espasmos em flexão da primeira infância e oligofrenia.

É fundamental o conceito de evolução, ou seja, a história natural da hipsarritmia, pois esta surge, tanto em crianças prèviamente sadias como em encefalopatas e modifica-se em vários sentidos, especialmente sob o aspecto eletrográfico.

Os fatôres etiológicos são os mesmos encontrados na gênese das encefalopatias infantis. Todavia, o que parece determinar a instalação do quadro hipsarrítmico é a condição inerente ao próprio encéfalo acometido. Na moderna conceituação da patogenia, os autores são unânimes em considerar o processo intimamente relacionado à maturação cerebral.

O prognóstico é, em geral, mau. Todavia, casos há que evoluem surpreendentemente bem. Conquanto seja lícito julgar que à melhora eletrográfica corresponda paralela recuperação clínica, esta correlação nem sempre se observa.

O tratamento é difícil e os resultados, muitas vêzes, incompletos ou transitórios. Não obstante a variabilidade dos resultados obtidos por diversos estudiosos e em que pese a transitoriedade de seus efeitos, o ACTH é o melhor agente terapêutico conhecido. Antibióticos e certos anticonvulsivantes são também usados, parecendo-nos, porém, que, muita vez, a medicação deva ser simplesmente sintomática. 


\section{RESUMO}

Resumindo as principais contribuições que resultaram na conceituação atual da hipsarritmia, destacam os autores a noção de que não constitui ela uma entidade clínica determinada, mas uma sindrome eletroclínica especial, própria da primeira infância, e cujo diagnóstico só pode ser firmado à luz do traçado eletrencefalográfico característico.

Analisam a variedade de fatôres etiológicos à maneira do que ocorre com as encefalopatias infantis em geral, chamando a atenção para a importância do fator etário, das condições traumáticas e do problema da anóxia cerebral neonatorum. Necessária ênfase é conferida ao fenômeno da maturação encefálica que explica, segundo o consenso dos autores modernos, as características peculiares da sindrome.

A hipsarritmia pode instalar-se em indivíduo previamente são ou em encefalopata, tendo evolução variável, pois tanto pode evolver favoràvelmente, o que é mais raro, como modificar-se, seja no sentido da epilepsia focal ou outra forma de epilepsia, o que é mais comum, ou, então, permanecer inalterada. Essa evolução - a história natural da hipsarritmia - é apreciada especialmente sob o aspecto eletrencefalográfico, o qual pode, ou não, ser acompanhado de correspondente evolução clínica.

A sintomatologia é variável, sendo de notar a grande dominância das crises de espasmos em flexão.

Sendo pobre o subsídio anátomo-patológico da condição, os autores, a êsse propósito, apenas acrescentam os dados referentes à necropsia de um dos seus casos, com achados inespecíficos.

O prognóstico, na maioria das vêzes mau, pode variar, especialmente após o uso do ACTH. O tratamento é difícil, freqüentemente desapontador. As melhores respostas são obtidas com o ACTH e, algumas vêzes, com os antibióticos e medicação sintomática, porém, quase sempre reaparece a sintcmatclogia, se suprimida a medicação que, por outro lado, nem sempre pode ser continuada.

\section{SUMMARY}

Hypsarhythmia. Report of 20 cases

Summarizing the main contributions which resulted in modern conception the authors point out that hypsarhythmia does not constitute a clinical entity but an electro-clinical syndrome, usually occurring in early childhood, whose diagnosis can only be made on the basis of characteristic electroencephalographic records. The authors analyse the etiological factors as they generally occur in the infantile encephalopaties and call attention to the etary factor, traumatic conditions, as well as the problem of neonatorum cerebral anoxia. Emphasis is given to the phenomenon of cerebral maturation which explains the peculiar characteristics of the syndrome. 
Hypsarhythmia may develop in a previously healthy individual or in an encephalopath. Its evolution is variable since it may evolve favorably, a rare occurrence, or may change either to a focal or some other type of epilepsy which is more common, or it may remain unaltered. This evolution - the natural history of hypsarhythmia - is considered specially from the electroencephalographic standpoint, being or not accompanied by the corresponding clinical evolution.

The symptomatology is variable, and it should be noted that the spasms in flexion are predominant.

The anatomo-pathological data are scarce; the authors only add some details referring to the autopsy of one of their cases, without any specific data.

The prognosis, mostly unfavorable, may change specially after the use of ACTH. The treatment is a difficult and frequently disappointing one. The best results are obtained with $\mathrm{ACTH}$ and, sometimes, with antibiotics and symptomatic medication but, almost always, when medication is discontinued, the symptomatology recurs. On the other hand, it is not always possible to continue with the medication.

\section{REFERENCIAS}

1. BOWER, B. D. \& JEAVONS, M. B. - Infantile spasms and hypsarhytmia. Lancet, 1959. 2. CHAO, D.; DRUCKMAN, R. \& KELlaWAY, P. - Convulsive Disorders of Children. W. B. Saunders Co., Filadélfia-Montreal, 1958. 3. COURJON, J. \& COTTE - L'épilepsie avant trois ans. Rév. Neurol., 99:68-76, 1958. 4. DRUCKMAN, R. \& CHAO, D. - Massive spasms in infancy and childhood. Epilepsia 4:61, 1955. 5. GASTAUT, H.; MIRIBEL, G.; FAVEL, P. \& VIGOUROUX, M. - Effets cliniques et électroencephalographiques de l'ACTH dans des différents types d'epilepsie. Rév. Neurol., 101:753-762, 1959. 6. GASTAUT, H. \& ROGER, A. - Étude électroencephalographique des convulsions infantiles. Pediatrie 8:603-614, 1953.7 . GASTAUT, H.; SALTIEL, J.; RAYBAUD, D.; PITOT, M. \& MEYNADIER, A. - A propos du traitment par l'ACTH des encéphalites myocloniques de la première enfance avec hypsarythmie. Pediatrie $14: 35-41$, 1958. 8. GIBBS, F. A. \& GIBBS, E. L. - Atlas of Electroencephalography, vol. II, págs. 24-30. Addison-Wesley Press Inc., Cambridge, Mass., 1952. 9. GIBBS, E. L.; FLEMING, M. M. \& GIBBS, F. A. - Diagnosis and prognosis of hypsarhythmia and infantile spasms. Pediatries 13: 66-73, 1954. 10. HESS, R., Jr. \& NEUHAUS, Th. - Das Elektrencephalogram bei Blitz-Nick und Salaamkrämpfen und beiandern Anfallsformen des Kindesalters. Arch. f. Psychiat. Neurol., 189:37-38, 1952. 11. KELlAWAY, P. - Neurologic status of patients with hypsarhythmia. In Molecules and Mental Health, ed, por F. A. Gibbs. J. B. Lippincott Co., Filadélfia-Montreal, 1959, págs. 134-149. 12, LAUNAY, C.; BLANC, C. \& REBUFFAT-DESCHAMPS, M. - Spasmes en flexion et hypsarythmie. Mise au point à propos de six observations personnelles. Presse Méd., 67:887-890, 1959. 13. LIVINGSTON, S.; EISNER, W. \& PAULI, R. - Minor motor epilepsy: diagnosis, treatment, prognosis. Pediatries 21:916-928, 1958. 14. LOW, N. - Treatment of hypsarhythmia with ACTH and cortisone. In Molecules and Mental Health. Ed. por F. A. Gibbs. J. B. Lippincott Co., Filadélfia-Montreal, 1959, págs. 124-133. 15. MAIA, J. A. \& LINS, S. G. - Contribuição ao estudo eletroclinico das hipsarritmias. Neurobiologia 22/23/24:64-101, 1959 a 1961. 16. NERY, O. \& MELLO, S. - Hipsarritmia. I Reunião Acad. Bras. Neurol., Bol. no 4, págs. 3-4, 1964. 17. OLIVEIRA, C. \& FERNANDES, I. - Hipsarritmia. Rev. Bras. Med., 18:368-376, 1961. PASSOUANT, P.; CADILHAC, J. \& RIBSTEIN, N. - Epilepsie et maturation cérébrale. Relatório apresentado ao XVII Congresso da Associacão dos.Pediatras de 
Lingua Francesa, Montpellier, 12-14 de outubro de 1959. 19. POSER, C. M. Neuropathologic findings in three cases of infantile spasms. In Molecules and Mental Health. Ed. por F. A. Gibbs. J. B. Lippincott Co., Filadélfia-Montreal, 1959, págs. 150-161. 20. ROBERTS, E. - Biochemical maturation of the central nervous system. In The Central Nervous System and Behavior, ed. por Mary A. B. Brazier, Madison Printing Co. Inc., Madison, N.J., 1960, págs. 127-186. 21. SABouraud, O.; COUTEL, V. \& DAvOST, P. H. - Evolution épileptique et démentielle avec tracé d'hysarythmie chez un enfant de 7 ans. Guérison clinique par l'ACTH. Rév. Neurol., 106:453-459, 1962. 22. SAMSON-DOLLFuS, D. - Aspect électroclinique de crises enregistrées chez des enfants présentant un tracé d'hypsarythmie. Rév. Neurol,, 99:126-132, 1958. 23. SOREL, L. \& DESAUSY-BAULOYE, A. - A propos de 21 cas d'hypsarythmie de Gibbs; son traitment spectaculaire par l'ACTH. Acta Neurol. Psychiat. Belg., 58:130-141, 1958. 24. STAMPS, F.; GIBBS, E. L.; GIBBS, F. A. \& ROSENTHAL, I. - Experience with ACTH treatment of hypsarhythmia. In Molecules and Mental Health, ed. por F. A. Gibbs. J. B. Lippincott Co., Filadélfia-Montreal, 1959, págs. 121-123. 25. STAMPS, F. W.; GIBBS, E. L. \& HAASE, E. - Epileptic patients treated with aureomycin. Dis. Nerv. System 2:227, 1951. 26. THIÉBAUT, F.; SACREZ, R.; ROHMER, F. \& ISCH-TREUSSARD, C. Corrélations électrocliniques dans 25 cas d'hypsarythmie de Gibbs. Rév. Neurol., 93:455-460, 1955. 27. WES, W. J. - On a peculiar form of infantile convulsion. Lancet 1:724, 1841.

Instituto de Neurologia da Universidade do Brasil - Av. Wenceslau Braz, 95, Botafogo - Rio de Janeiro, GB, Biasil. 